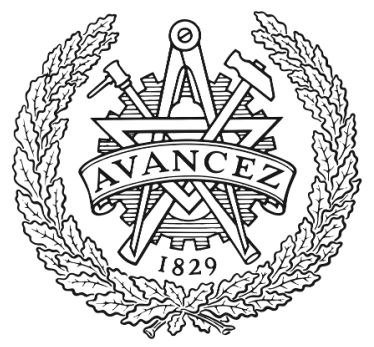

CHALMERS

UNIVERSITY OF TECHNOLOGY

\title{
Study of the parametric dependence of linear and nonlinear microtearing modes in conventional tokamak discharges
}

Downloaded from: https://research.chalmers.se, 2023-04-26 08:57 UTC

Citation for the original published paper (version of record):

Rafiq, T., Kritz, A., Weiland, J. et al (2018). Study of the parametric dependence of linear and nonlinear microtearing modes in conventional

tokamak discharges. Physics of Plasmas, 25(1). http://dx.doi.org/10.1063/1.5009105

N.B. When citing this work, cite the original published paper. 


\title{
Study of the parametric dependence of linear and nonlinear microtearing modes in conventional tokamak discharges
}

\author{
T. Rafiq, ${ }^{1, a)}$ A. H. Kritz, ${ }^{1}$ J. Weiland, ${ }^{2}$ L. Luo, ${ }^{1, b)}$ and E. Schuster ${ }^{3}$ \\ ${ }^{1}$ Department of Physics, Lehigh University, Bethlehem, Pennsylvania 18015, USA \\ ${ }^{2}$ Chalmers University of Technology and EURATOM-VR Association, Gothenburg, Sweden \\ ${ }^{3}$ Department of Mechanical Engineering and Mechanics, Lehigh University, Bethlehem, Pennsylvania 18015, \\ USA
}

(Received 12 October 2017; accepted 19 December 2017; published online 8 January 2018)

A reduced transport model for microtearing modes is developed for use in integrated predictive modeling studies, employing a unified fluid/kinetic approach to derive the nonlinear dispersion relation. This approach advances the kinetic description and allows the inclusion of nonlinear effects due to magnetic fluctuations. In this numerical study, the dependence of the microtearing mode real frequency and growth rate on plasma parameters and on DIII-D like L-mode and Hmode plasma profiles is examined. The magnetic fluctuation strength as well as electron thermal diffusivity due to microtearing modes is computed. The saturated amplitude of the magnetic fluctuations is calculated utilizing numerically determined microtearing mode eigenvalues in the nonlinear microtearing modes envelope equation. It is found that the electron temperature gradient in the presence of moderate collision frequency is required for the microtearing mode to become unstable. The effects of small and large collisionality and small and large wavenumbers on microtearing modes are found to be stabilizing, while the effects of density gradient, plasma beta, low current density, and large magnetic shear are found to be destabilizing. The microtearing mode growth rate, magnetic fluctuation strength, as well as electron thermal diffusivity is found to be larger in the H-mode plasma than in the L-mode plasma. Published by AIP Publishing.

https://doi.org/10.1063/1.5009105

\section{INTRODUCTION}

Microtearing modes are short wavelength ion scale electromagnetic instabilities driven by electron temperature gradient in the collisional regime. ${ }^{1,2}$ These modes have been identified as a source of significant electron thermal transport in tokamak discharges. For example, microtearing modes have been predicted to be unstable in high- $\beta$ spherical discharges, MAST ${ }^{3-5}$ and $\mathrm{NSTX}^{6-13}$ and in standard tokamak discharges, DIII-D, ${ }^{14,15}$ Alcator C-Mod, ${ }^{16}$ JET $^{17}$ and ASDEX Upgrade. ${ }^{18}$ The existence of microtearing modes in tokamak discharges highlights the need to determine the electron thermal transport due to the nonlinear saturated microtearing mode. In the previous publications, ${ }^{2,7,8,19,20}$ the ratio of magnetic fluctuations, associated with microtearing modes, to magnetic field are assumed to be approximately equal to the ratio of electron gyroradius to the electron temperature gradient scale length. This assumption is based on mixing length estimate which provides a reasonable estimate for the saturated amplitude for temperature gradients that are sufficiently greater than the linear threshold. However, the mixing length estimate does not capture the dependence of the microtearing mode contribution to electron thermal transport on other parameters such as collisionality, plasma $\beta_{\mathrm{e}}$, magnetic-q, magnetic shear, and mode wavenumber. Therefore, transport models that rely on the simplified saturation estimate are incapable of reproducing the correct scaling dependence on these

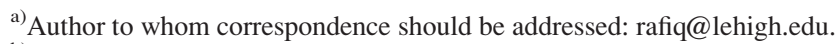

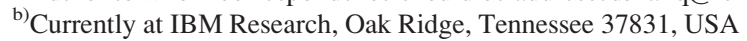

parameters, which illustrates the need for an improved saturation model. In order to understand how microtearing modes affect transport and, consequently, the evolution of electron temperature in tokamak discharges, a reduced transport model has been developed ${ }^{21}$ for use in integrated predictive modeling studies. ${ }^{22}$ The electron momentum and density equations, Maxwell's equations, Ampere's law, and quasi-neutrality condition were employed in the development. A unified fluid/ kinetic approach was used which included nonlinear effects due to electrostatic and magnetic fluctuations, as well as effects due to collisionality, electron temperature and density gradients, and arbitrary curvature. The influence of third order nonlinear effects on a multi-wave system were considered, and the third order effects provided a possible mechanism for the temporal saturation of the microtearing instability. The envelope equation for the nonlinear microtearing modes was introduced, which provided the initial linear growth and the final stabilization, and was used to calculate the saturation level of the nonlinear microtearing instability. An iterative approach was applied to calculate the nonlinear distribution function, which, in turn, was used to calculate the nonlinear parallel current in obtaining a nonlinear dispersion relation. The resulting dispersion relation, developed in Ref. 21 , is included in Sec. II and is utilized in the study of the dependence of microtearing electron thermal transport on plasma parameters. In this paper, dependencies of the fastest growing microtearing mode on collisionality, $\beta_{\mathrm{e}}$, magnetic shear, electron density and temperature normalized gradient are illustrated. The effects of magnetic fluctuations on microtearing 
mode real frequency and growth rate are also investigated. The paper is organized as follows: In Sec. II, the microtearing mode anomalous transport model, which has been developed and described in Ref. 21, is briefly summarized both for the low collisionality and collision dominated cases. The dispersion relation that results and that can be used to compute the growth rate (and associated real frequency) of the most unstable microtearing mode is presented. In Sec. III A, the dependence of growth rate and real frequency of the most unstable microtearing mode on plasma wavenumber, collision frequency, temperature gradient, magnetic-q, magnetic shear, density gradient, and plasma beta are illustrated for a DIII-D like L-mode discharge. The dependence of the tearing mode real frequency, growth rate, magnetic fluctuation, and electron thermal diffusivity on DIII-D L-mode and H-mode plasma is also shown in Sec. III. The effects of microtearing modes on electron thermal transport in DIII-D discharges are summarized in Sec. IV.

\section{MICROTEARING MODE ANOMALOUS TRANSPORT MODEL}

With the effect of collisions introduced in the term on the left side, the nonlinear microtearing dispersion relation developed in Ref. 21 is

$$
\begin{gathered}
{\left[\omega+\alpha_{0} \frac{m_{\mathrm{e}}}{m_{\mathrm{i}}} \frac{k_{\mathrm{y}}^{2} \rho_{s}^{2}}{\beta_{\mathrm{e}}}\left(\omega-\omega_{\mathrm{De}}+i \nu_{\mathrm{ei}}\right)\right]=\omega_{* \mathrm{e}}\left(1+\eta_{\mathrm{e}}\right)} \\
-\alpha_{1} \frac{\eta_{\mathrm{e}} \omega_{* e}\left(\omega-\omega_{\mathrm{De}}\right)}{\omega-\omega_{\mathrm{De}}+i \nu_{\mathrm{ei}}}-\frac{\omega_{* \mathrm{e}}-\omega_{\mathrm{De}}}{\omega-\omega_{\mathrm{De}}+i \nu_{\mathrm{ei}}} \frac{k_{\|}^{2} u_{A}^{2}}{\omega} \\
-\alpha_{2} \eta_{\mathrm{e}} \sum_{k^{\prime}} \frac{\omega_{* \mathrm{e}}-\omega_{* \mathrm{e}}^{\prime}}{\omega_{k^{\prime}+k}-\omega_{\mathrm{Dek}^{\prime}+\mathrm{k}}} \frac{\frac{1}{2}\left|\frac{\delta \mathbf{B}_{k^{\prime}}}{B} \cdot \mathbf{k} u_{\mathrm{th}}\right|^{2}}{\omega_{k+k^{\prime}}-\omega_{\mathrm{Dek}+\mathrm{k}^{\prime}}+i \nu_{\mathrm{ei}}} .
\end{gathered}
$$

The nonlinear microtearing mode dispersion relation in Eq. (1) is applicable for the collisionless, the semi-collisional and the collisional regimes. In Eq. (1), $\omega$ is the mode eigenfrequency; $m_{\mathrm{e}}$ is the electron mass; $m_{\mathrm{i}}$ is the ion mass; $k_{\mathrm{y}}$ is the wave vector in the poloidal direction; $\rho_{s} \equiv c_{s} / \Omega_{i}$ is the ion Larmor radius with the electron (rather than the ion) temperature; $c_{s} \equiv \sqrt{T_{e} / m_{i}}$ is the speed of sound; $\Omega_{i}=e B / m_{i}$ is the ion gyrofrequency; $\beta_{\mathrm{e}}=\mu_{0} n_{e} T_{e} / B^{2}$ is the ratio of the electron pressure to the magnetic pressure; $\mu_{0}$ is the permeability of free space; $n_{\mathrm{e}}$ is the electron density; $T_{\mathrm{e}}$ is the electron temperature; $B$ is the magnetic field intensity; and $\delta B$ is the magnetic fluctuation.

The magnetic drift frequency, $\omega_{\text {De }}$, for microtearing modes can be written in terms of an average curvature

$$
\omega_{\mathrm{De}}=\frac{2 k_{y} \rho_{\mathrm{s}} c_{\mathrm{s}}}{R} \frac{r}{R}\left(1-\frac{1}{q^{2}}\right),
$$

where $R$ is the major radius to geometric center of the flux surface, $r$ is the distance from the geometric center to the flux surface, and $q$ is the safety factor. The electron diamagnetic drift frequency, $\omega_{* e}$, is given by

$$
\omega_{* e}=k_{y} T_{e} g_{\text {ne }} / e B R .
$$

The ratio of the electron temperature gradient, $g_{\mathrm{Te}} \equiv-R \hat{\mathbf{x}} \cdot \nabla T_{e} / T_{e}$, and the electron density gradient, $g_{\text {ne }} \equiv-R \hat{\mathbf{x}} \cdot \nabla n_{e} / n_{e}$, is represented by $\eta_{e}$, where

$$
\eta_{e} \equiv g_{\mathrm{Te}} / g_{\mathrm{ne}}=\frac{n_{e} \hat{\mathbf{x}} \cdot \nabla T_{e}}{T_{e} \hat{\mathbf{x}} \cdot \nabla n_{e}}
$$

and where the unit vector $\hat{\mathbf{x}}$ is in the radial direction.

The electron collision frequency $\nu_{\mathrm{ei}}$ is given by

$$
\nu_{\mathrm{ei}}=4(2 \pi)^{1 / 2} n_{e}(\ln \lambda) e^{4} Z_{\mathrm{eff}} /\left[3\left(4 \pi \epsilon_{o}\right)^{2} m_{e}^{1 / 2}\left(k_{b} T_{e}\right)^{3 / 2}\right],
$$

where $\ln \lambda$ is the Coulomb logarithm, $Z_{\text {eff }}$ is the effective charge, $\varepsilon_{0}$ is the permittivity of free space, and $k_{b}$ is the Boltzmann constant. The parallel propagation vector is given by

$$
k_{\|}=\frac{2}{L_{\mathrm{s}}} \frac{k_{\mathrm{y}}^{2}}{k_{\mathrm{x}}^{2}}
$$

where $L_{\mathrm{s}}=R q / \hat{s}$ is the magnetic shear length with the magnetic shear given by $\hat{s}=(r / q)(d q / d r)$.

The Alfvén velocity in Eq. (1) is $u_{A}=B / \sqrt{\mu_{0} n_{e} m_{i}}$, and the electron thermal velocity is $u_{\mathrm{th}} \equiv \sqrt{T_{e} / m_{e}}$. The last term on the RHS of Eq. (1) is the nonlinear term in which

$$
\omega_{k+k^{\prime}}=\omega_{k}+\omega_{k^{\prime}} .
$$

In Sec. II A, the dispersion relation is examined in the limit of $\nu_{\mathrm{ei}} \gg \omega$ in order to determine $\alpha_{0}, \alpha_{1}$, and $\alpha_{2}$, which appear in Eq. (1). These factors are obtained by comparing Eq. (3) (below) with kinetic Eq. (8) in Ref. 2.

\section{A. High collisionality limit, $v_{\mathrm{ei}} \gg \omega$}

In the collision dominant limit, the nonlinear microtearing mode dispersion relation takes the form

$$
\begin{aligned}
& {\left[\omega+i \alpha_{0} \frac{m_{\mathrm{e}}}{m_{\mathrm{i}}} \frac{k_{\mathrm{y}}^{2} \rho_{s}^{2}}{\beta_{\mathrm{e}}} \nu_{\mathrm{ei}}\right]} \\
& =\omega_{* \mathrm{e}}\left(1+\eta_{\mathrm{e}}\right)+i \alpha_{1} \frac{\eta_{\mathrm{e}} \omega_{* e}\left(\omega-\omega_{\mathrm{De}}\right)}{\nu_{\mathrm{ei}}}+i \frac{\omega_{* \mathrm{e}}-\omega_{\mathrm{De}}}{\nu_{\mathrm{ei}}} \frac{k_{\|}^{2}}{\omega} \\
& -i \alpha_{2} \eta_{\mathrm{e}} \frac{u_{\mathrm{th}}^{2}}{\nu_{\mathrm{ei}}} \sum_{k^{\prime}} \frac{1}{2}\left|\frac{\delta \mathbf{B}_{k^{\prime}}}{B} \cdot \mathbf{k}\right|^{2} \frac{\omega_{* \mathrm{e}}-\omega_{* \mathrm{e}}^{\prime}}{\omega_{k}-\omega_{\mathrm{Dek}}+\omega_{k^{\prime}}-\omega_{\mathrm{Dek}^{\prime}}}
\end{aligned}
$$

When $\omega_{\text {De }}=0$ and $k_{\|}=0$, the fluid dispersion relation in Eq. (3) is similar to the kinetic dispersion relation given in Eq. (8) in Ref. 2. The parameters $\alpha_{0}, \alpha_{1}$, and $\alpha_{2}$ are obtained by comparing Eqs. (3) and (8) in Ref. 2

$$
\alpha_{0}=\frac{3 \sqrt{\pi}}{8}, \quad \alpha_{1}=\frac{3}{8} \Gamma\left(\frac{9}{2}\right), \quad \text { and } \quad \alpha_{2}=\frac{5}{6} \Gamma\left(\frac{11}{2}\right) .
$$

Note: the numerical values of $\alpha_{0}, \alpha_{1}$, and $\alpha_{2}$ depend on the choice of collision model. The values of the three $\alpha^{\prime} s$ above correspond to the Lorentz collision model used in Ref. 2 . The numerical values of the $\alpha^{\prime} s$ change if a different collision model is employed. 


\section{B. Calculation of magnetic fluctuation and thermal diffusivity}

The linear growth rate will dominate initially but will saturate when the growth rate becomes sufficiently large. The saturation level is determined by examining the microtearing mode envelope equation

$$
\begin{aligned}
\frac{\partial \hat{A}}{\partial t}= & \frac{3}{8} \Gamma\left(\frac{9}{2}\right) \frac{\eta_{\mathrm{e}} \omega_{* e}\left(\omega-\omega_{\mathrm{De}}\right)}{\nu_{\mathrm{ei}}} \hat{A}-\frac{5}{6} \Gamma\left(\frac{11}{2}\right) \eta_{\mathrm{e}} \frac{u_{\mathrm{th}}^{2}}{\nu_{\mathrm{ei}}} \\
& \times \sum_{k^{\prime}}\left|\frac{\delta \mathbf{B}_{k^{\prime}}}{B} \cdot \mathbf{k}\right|^{2} \frac{\omega_{* \mathrm{e}}-\omega_{* \mathrm{e}}^{\prime}}{\omega_{k}-\omega_{\text {Dek }}+\omega_{k^{\prime}}-\omega_{\mathrm{Dek}^{\prime}}} \hat{A} .
\end{aligned}
$$

The last term in Eq. (4) is a cubic nonlinear term, which enters as a nonlinear frequency shift. The harmonic oscillations cancel due to the term that is proportional to $\left|\delta \mathbf{B}_{k^{\prime}} \cdot \mathbf{k}\right|^{2}$. Therefore, Eq. (4) is a nonlinear microtearing mode dispersion relation written as an envelope equation in time. The envelope equation can be used to calculate the saturation level of the microtearing instability. The solution of Eq. (4) will provide the initial linear growth and the final stabilization. In order to solve Eq. (4), in principle, the spectrum of magnetic fluctuations is needed. However, similar to the treatment of drift waves, the use of the most unstable linear growing microtearing mode is sufficient.

The nonlinear term in both Eqs. (1) and (4) scales as $k^{2} \chi_{\mathrm{e}}$, where $\chi_{\mathrm{e}}$, the electron thermal diffusivity due to microtearing mode instabilities, is given by

$$
\chi_{\mathrm{e}}=\frac{u_{\mathrm{th}}^{2}}{\nu_{\mathrm{ei}}} \frac{|\delta B|^{2}}{B^{2}} .
$$

The $|\delta B|^{2} / B^{2}$ in Eq. (5) can be computed using the nonlinear microtearing mode envelope equation [Eq. (4)]. Note that in Ref. 2, $|\delta B| / B$ is approximated as $\rho_{\mathrm{e}} / L_{\mathrm{Te}}$. However, the computation of $|\delta B| / B$ in Eq. (4) will depend upon the most unstable eigenvalue as well as on its sideband in the $k_{\mathrm{y}}$ spectrum.

\section{NUMERICAL RESULTS}

\section{A. Dependence of microtearing modes eigenvalues on plasma parameters}

A selection of results are presented in this section in which linear microtearing mode eigenvalues are computed by ignoring nonlinear term in Eq. (1). The dependence on the wavenumber, $k_{\mathrm{y}} \rho_{\mathrm{s}}$, of the normalized growth rate $\left(R \gamma / c_{s}\right)$ of the most unstable microtearing mode and the normalized microtearing mode real frequency $\left(R \omega / c_{s}\right)$, associated with the most unstable mode, is shown in Fig. 1 for a selection of normalized electron temperature gradient [defined in Eq. (2)], $\eta_{e}=2.5,5.0$, and 10. The other plasma parameter values correspond to a DIII-D L-mode like discharge are $R=1.68 \mathrm{~m}, r_{\text {min }}=0.50 \mathrm{~m}, \quad q=3.5, B_{0}=1.6 \mathrm{~T}, n_{\mathrm{e}}=2.65$ $\times 10^{19} \mathrm{~m}^{-3}, \quad T_{\mathrm{e}}=0.4 \mathrm{keV}, \quad Z_{\text {eff }}=1.46, g_{\mathrm{Te}}=19.4, \quad g_{\text {ne }}$ $=3.91, \beta_{\mathrm{e}}=0.01 k_{\mathrm{x}}=10 k_{\mathrm{y}}$, and $\nu_{\mathrm{ei}}=2.03 \times 10^{5} \mathrm{~s}^{-1}$.

It is shown in Fig. 1(a) that the maximum growth rate of the most unstable microtearing mode occurs at $k_{\mathrm{y}} \rho_{\mathrm{s}} \approx 0$.1. It is also shown that the maximum growth rate increases as the electron temperature gradient increases, indicating that these modes are driven by the electron temperature gradient. Since the maximum growth rate occurs for $k_{\mathrm{y}} \rho_{\mathrm{s}} \approx 0.1$, the microtearing modes do not have the scale of electron temperature gradient modes. Instead, the microtearing modes are a short wavelength ion scale modes and are likely to coexist with ion temperature gradient modes in tokamak discharges. In addition, the microtearing modes are electromagnetic modes since they result from electromagnetic effects included in the derivation of the microtearing mode model.

In Fig. 1(b), it is shown that the microtearing mode real frequency associated with the most unstable mode increases with an increasing electron temperature gradient. The positive sign of the real frequency indicates that the microtearing modes propagate in the electron diamagnetic drift direction. The microtearing modes are found to be stabilized for both shorter and longer wavelengths due to the effects of field line bending included in the first term in Eq. (1). The bending effects are found to be stronger at large values of $\eta_{\mathrm{e}}$ resulting in the cut-off in the microtearing mode growth rate occurring at lower values in the $k_{\mathrm{y}} \rho_{\mathrm{s}}$ spectrum. Consequently, large electron temperature gradients can stabilize the short wavelength microtearing modes.

In Fig. 2, the dependence of the microtearing mode growth rate of the most unstable mode and the associated real frequency is shown as a function of $k_{\mathrm{y}} \rho_{s}$ for three values of normalized density gradient, $g_{\text {ne }}$ (dashed curves for $g_{\text {ne }}=7.82$, solid lines for $g_{\text {ne }}=3.91$, and dashed-dotted curves for $\left.g_{\text {ne }}=1.95\right)$. The maximum growth rate and the associated real frequency are both found to increase with increasing density gradient. As noted earlier, the growth rate is found to peak in the vicinity of $k_{\mathrm{y}} \rho_{s}=0.1$. The cut-off in the growth rate of the microtearing modes occurs at larger values in the $k_{\mathrm{y}} \rho_{s}$ spectrum as the electron density gradient increases. This is in contrast with the cut-off occurring at lower values in the $k_{\mathrm{y}} \rho_{s}$ spectrum as the electron temperature gradient increases. Since the location of the largest growth rate of the most unstable microtearing mode remains at $k_{\mathrm{y}} \rho_{s} \approx 0.1$ in the $k_{\mathrm{y}} \rho_{s}$ spectrum, the location is relatively insensitive to the value of the electron temperature gradient, $\eta_{\mathrm{e}}$, and electron density gradient, $g_{\text {ne }}$.

The dependence of the growth rate of the most unstable microtearing mode and the associated frequency is shown in Fig. 3 as a function of normalized wavenumber for different values of collision frequency (dashed curves for $0.5 \nu_{\mathrm{ei}}$, solid curves for $\nu_{\mathrm{ei}}$, and dashed-dotted curves for $2.0 \nu_{\mathrm{ei}}$, where the magnitude of the collision frequency is $\nu_{\mathrm{ei}}=2.03 \times 10^{5} \mathrm{~s}^{-1}$ ). The maximum microtearing mode growth rate is found to decrease with increasing collision frequency. Also, it is seen that, as the collision frequency increases, both the most unstable mode and the cut-off occur at lower values of $k_{\mathrm{y}} \rho_{\mathrm{s}}$. For the range of collision frequencies considered, the real frequency, associated with the most unstable microtearing mode, is shown to have a weak dependence on collision frequency.

In Fig. 4, the dependence of microtearing mode growth rate of the most unstable mode and the associated frequency are presented as a function of normalized collision frequency $\left(R \nu_{\mathrm{ei}} / c_{\mathrm{s}}\right)$ for different values of normalized electron 

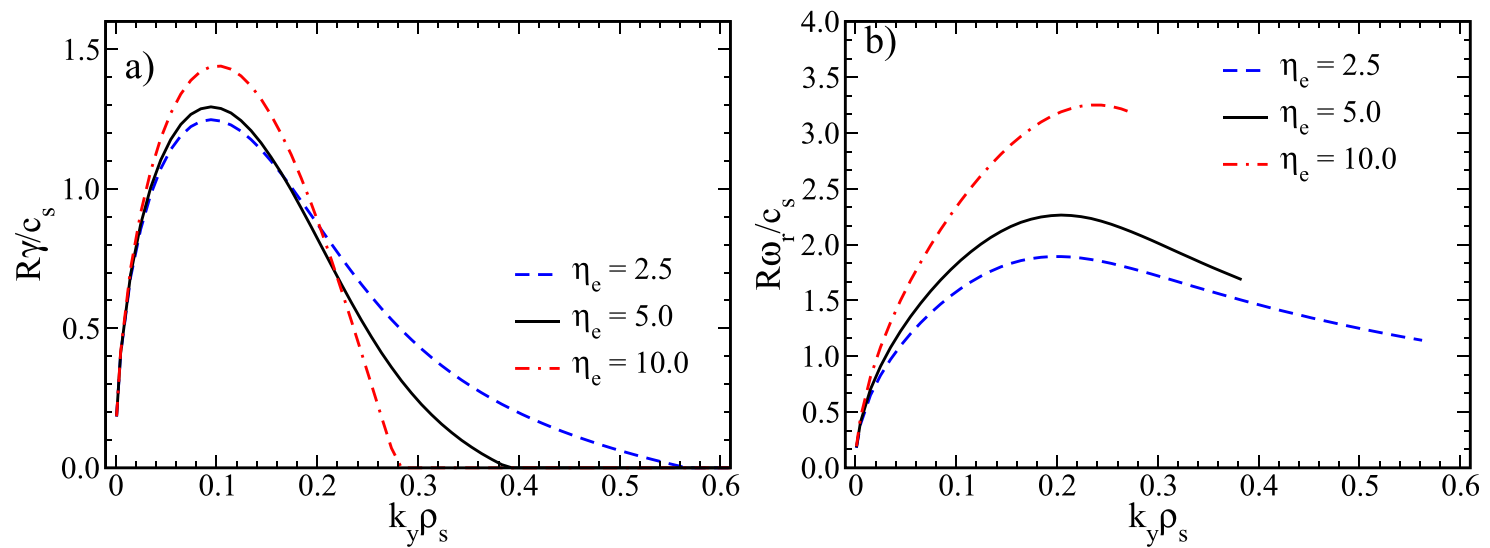

FIG. 1. (a) Normalized growth rate of the fastest growing mode and (b) the normalized real frequency, associated with that mode, as a function of $k_{\mathrm{y}} \rho_{s}$ for different values of $\eta_{\mathrm{e}}$ with $R=1.68 \mathrm{~m}, r_{\min }=0.50 \mathrm{~m}, q=3.5, B_{0}=1.6 \mathrm{~T}, n_{\mathrm{e}}=2.65 \times 10^{19} \mathrm{~m}^{-3}, T_{\mathrm{e}}=0.4 \mathrm{keV}, Z_{\mathrm{eff}}=1.46, g_{\mathrm{Te}}=19.4, g_{\mathrm{ne}}=3.91, \beta_{\mathrm{e}}=$ $0.01, k_{\mathrm{x}}=10 k_{\mathrm{y}}$, and $\nu_{\mathrm{ei}}=2.03 \times 10^{5} \mathrm{~s}^{-1}$.
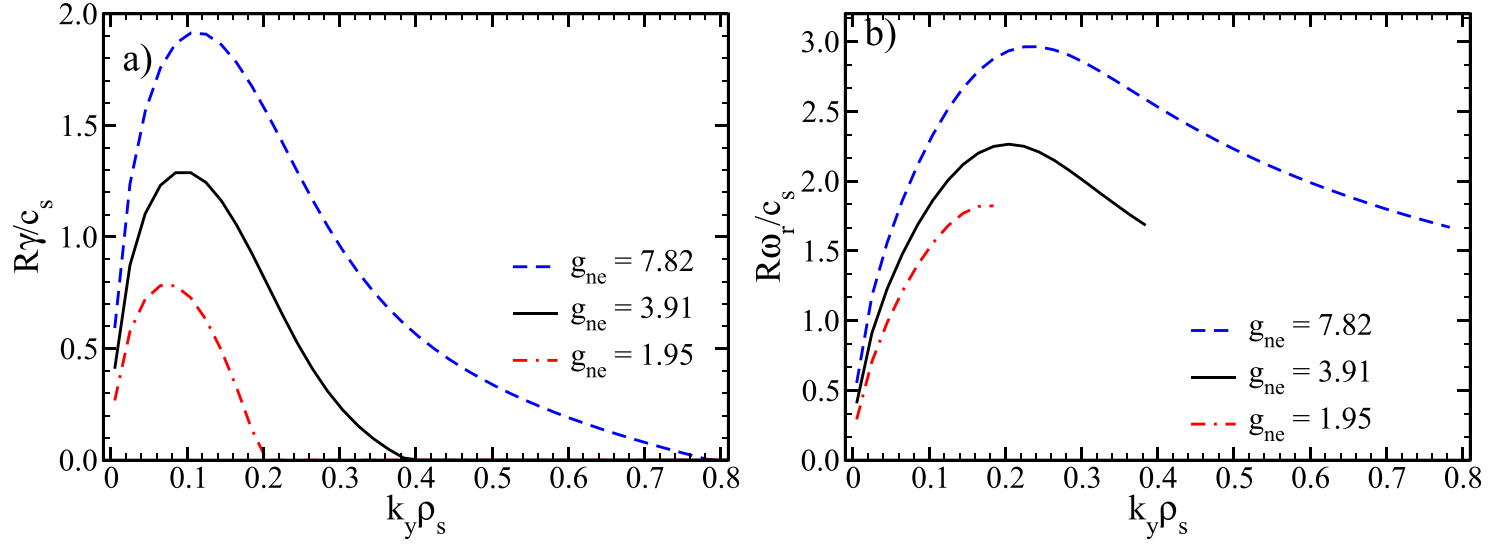

FIG. 2. (a) Normalized growth rate of the most unstable mode and (b) the associated normalized real frequency as a function of $k_{\mathrm{y}} \rho_{s}$ for different values of normalized density gradient $g_{\mathrm{ne}} \equiv-R \hat{\mathbf{x}} \cdot \nabla n_{e} / n_{e}$. The other plasma parameters are the same as those used in Fig. 1 .

temperature gradient. It is found that the microtearing mode growth rate and corresponding real frequency both increase as the electron temperature gradient increases. Also, the maximum growth rate occurs at a higher normalized collision frequency as the electron temperature gradient increases. Both small and large collisionality are found to be stabilizing.

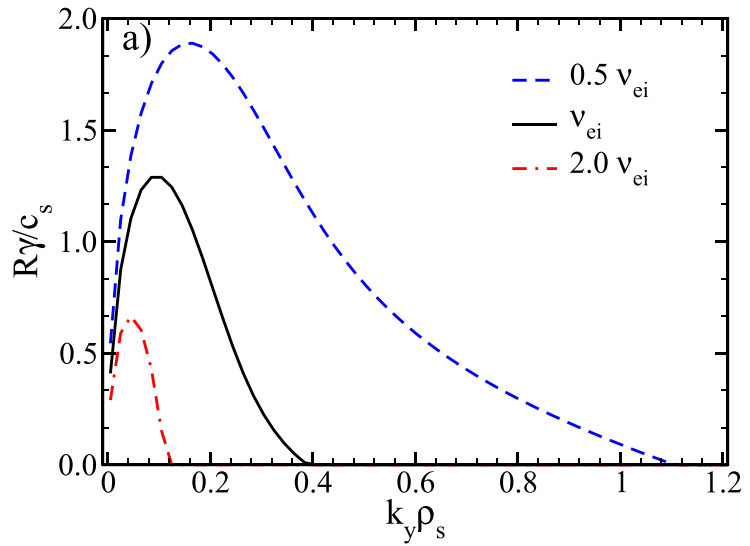

The growth rate of the most unstable microtearing mode and real frequency associated with that mode are plotted in Fig. 5 as a function of $\eta_{\mathrm{e}}$ for three values of electron collision frequency (dashed curves for $\nu_{\mathrm{e}}=0.2 \nu_{\mathrm{ei}}$, dotted-dashed curves for $\nu_{\mathrm{e}}=0.5 \nu_{\mathrm{ei}}$, and solid curves for $\nu_{\mathrm{e}}=\nu_{\mathrm{ei}}$, where $\left.\nu_{\mathrm{ei}}=2.03 \times 10^{5} \mathrm{~s}^{-1}\right)$. The destabilizing effect of increasing normalized electron temperature gradient, $\eta_{\mathrm{e}}$, is shown in

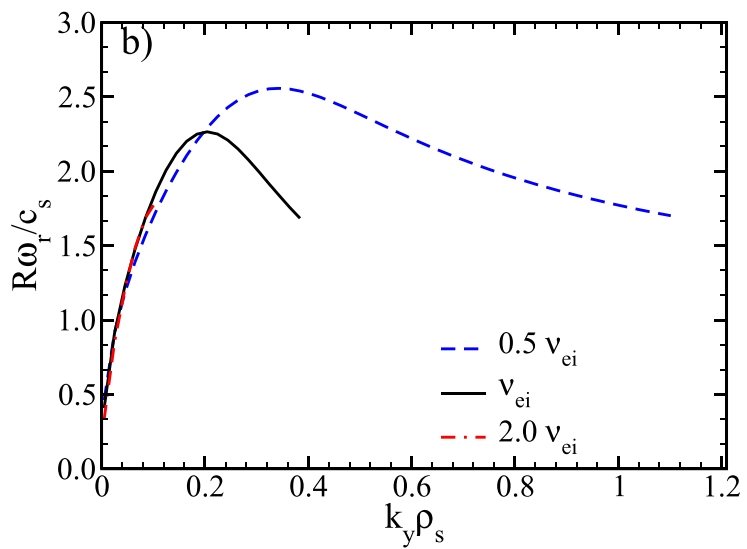

FIG. 3. (a) Normalized growth rate of the fastest growing microtearing mode and (b) the normalized real frequency as a function of $k_{\mathrm{y}} \rho_{s}$ for different values of collision frequency. The dashed curves are for $0.5 \nu_{\mathrm{ei}}$, the solid curves are for $\nu_{\mathrm{ei}}$, and the dashed-dotted curves are for $2.0 \nu_{\mathrm{ei}}$, where $\nu_{\mathrm{ei}}=2.03 \times 10^{5} \mathrm{~s}^{-1}$. The other plasma parameters are the same as those used in Fig. 1. 

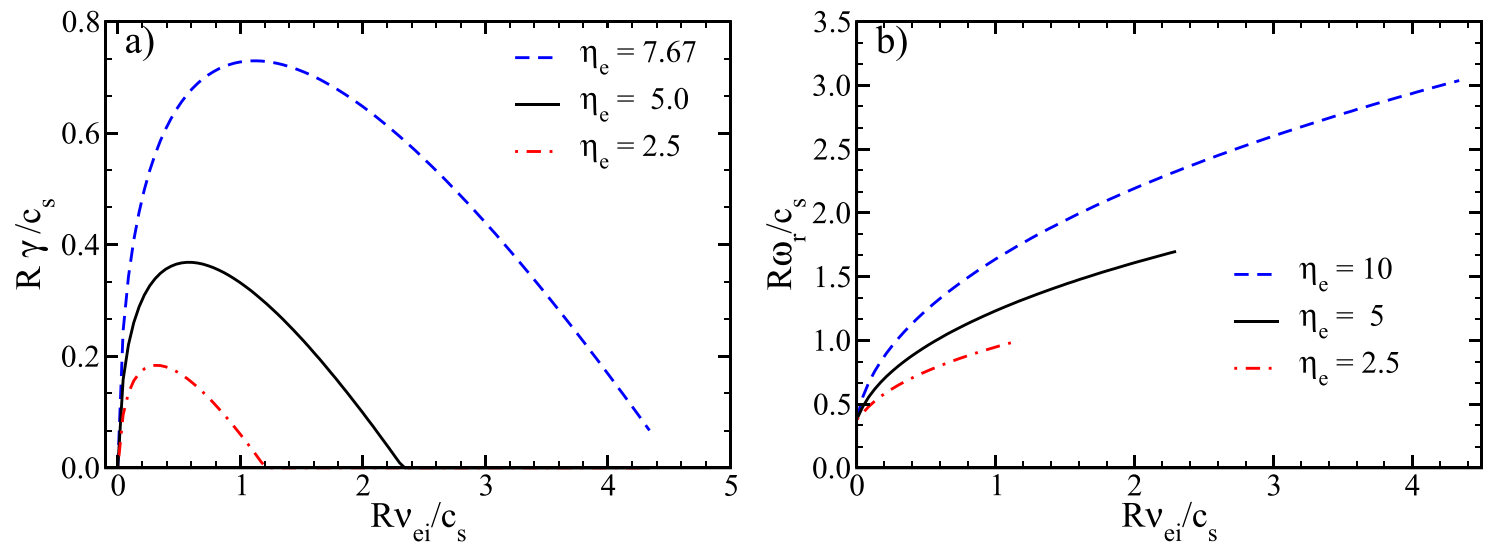

FIG. 4. (a) The normalized growth rate of the most unstable microtearing mode and (b) the normalized real frequency associated with that mode are plotted as a function of $R \nu_{\mathrm{ei}} / c_{\mathrm{s}}$ for different values of $\eta_{\mathrm{e}}$. For the results shown $k_{\mathrm{x}}=40 k_{\mathrm{y}}$. The other values are the same as those used in Fig. 1 .
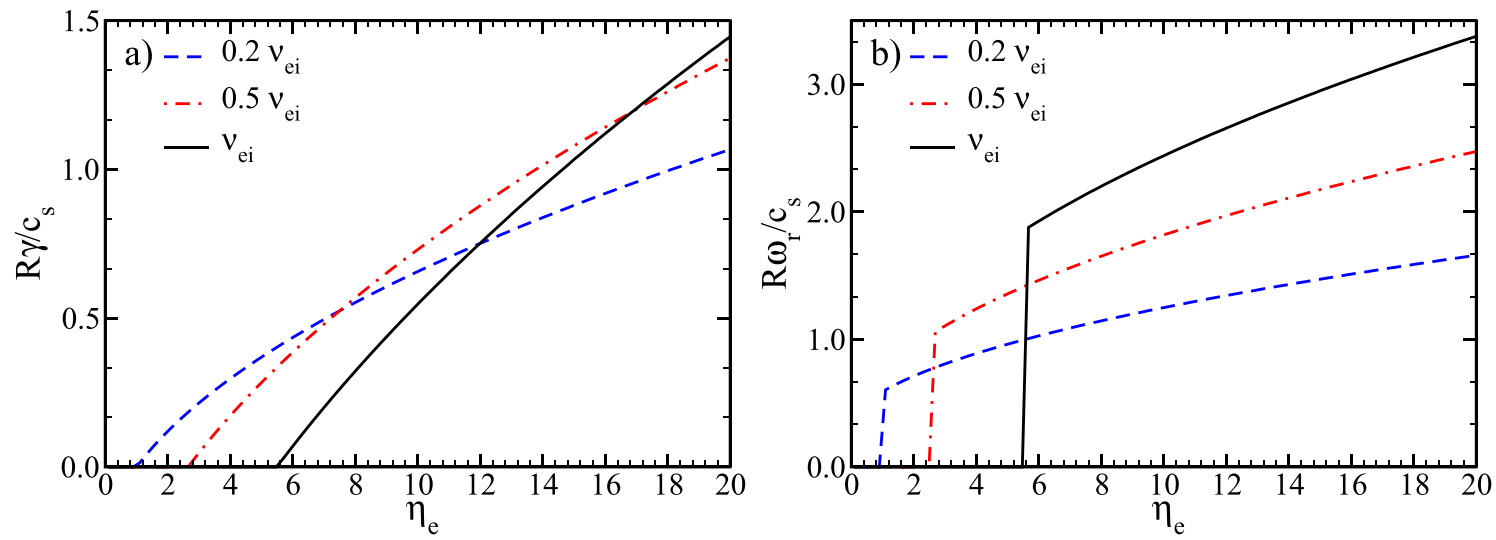

FIG. 5. (a) The dependence of the normalized growth rate of the most unstable microtearing mode and (b) the associated normalized frequency are shown as a function of the temperature gradient, $\eta_{\mathrm{e}}$. The dashed curves are for $0.2 \nu_{\mathrm{ei}}$, the dotted-dashed curves are for $0.5 \nu_{\mathrm{ei}}$, and the solid curves are for $\nu_{\mathrm{ei}}$ where $\nu_{\mathrm{ei}}=2.03 \times 10^{5} \mathrm{~s}^{-1}$. The plots are obtained with $k_{\mathrm{y}} \rho_{\mathrm{s}}=0.1$ and with $k_{\mathrm{x}} \rho_{\mathrm{s}}=4.0$. The other parameters used in obtaining the plots are the same as those used in Fig. 1.

Fig. 5. Also shown is that $\eta_{\mathrm{e}}>0$ is required for the microtearing mode instability and that the threshold in $\eta_{\mathrm{e}}$ increases as $\nu_{\mathrm{e}}$ increases.

For the most unstable microtearing mode, the normalized microtearing mode growth rate, $R \gamma / c_{\mathrm{s}}$ (solid line), and associated normalized microtearing mode real frequency, $R \omega / c_{\mathrm{s}}$ (dashed line), are shown as a function of normalized

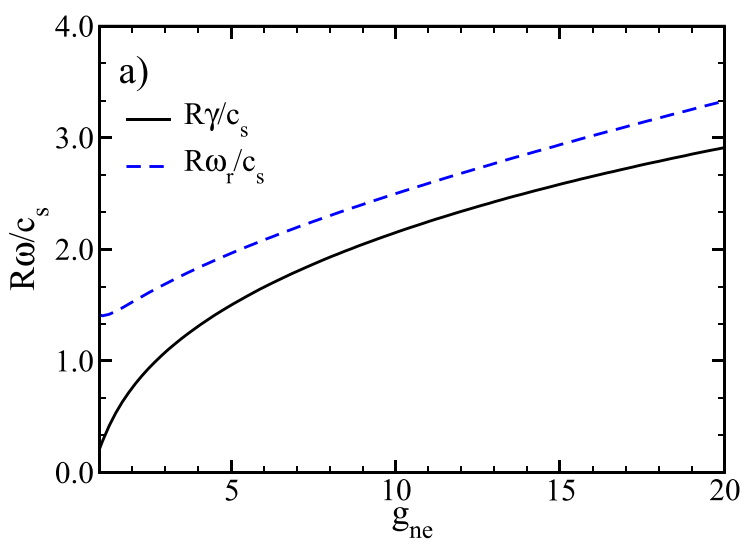

density gradient in Fig. 6(a) and as a function of plasma $\beta_{\mathrm{e}}$ in Fig. 6(b). The growth rate and real frequency both increase with increasing density gradient. When varying the normalized density gradient, $g_{\text {ne }}$, the normalized temperature gradient, $g_{\text {Te }}$, is kept fixed. The effects of density gradient on MTM modes are found to be destabilizing due to the inclusion of electrostatic potential perturbation in the MTM

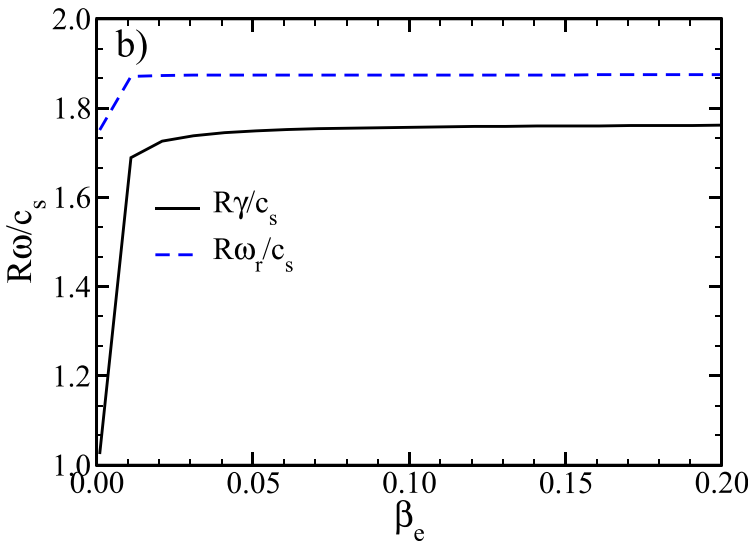

FIG. 6. (a) Normalized microtearing mode growth rate, $R \gamma / c_{\mathrm{s}}$ (solid line) and normalized microtearing modes real frequency, $R \omega / c_{\mathrm{s}}$ (dashed line) for the most unstable microtearing mode as a function of normalized density gradient, $g_{\text {ne }}$ and (b) as a function of plasma $\beta_{\mathrm{e}}$. For the result shown $k_{\mathrm{y}} \rho_{\mathrm{s}}=0.1$ and $k_{\mathrm{x}} \rho_{\mathrm{s}}=0.5$. The other parameters are the same as those used in Fig. 1. 

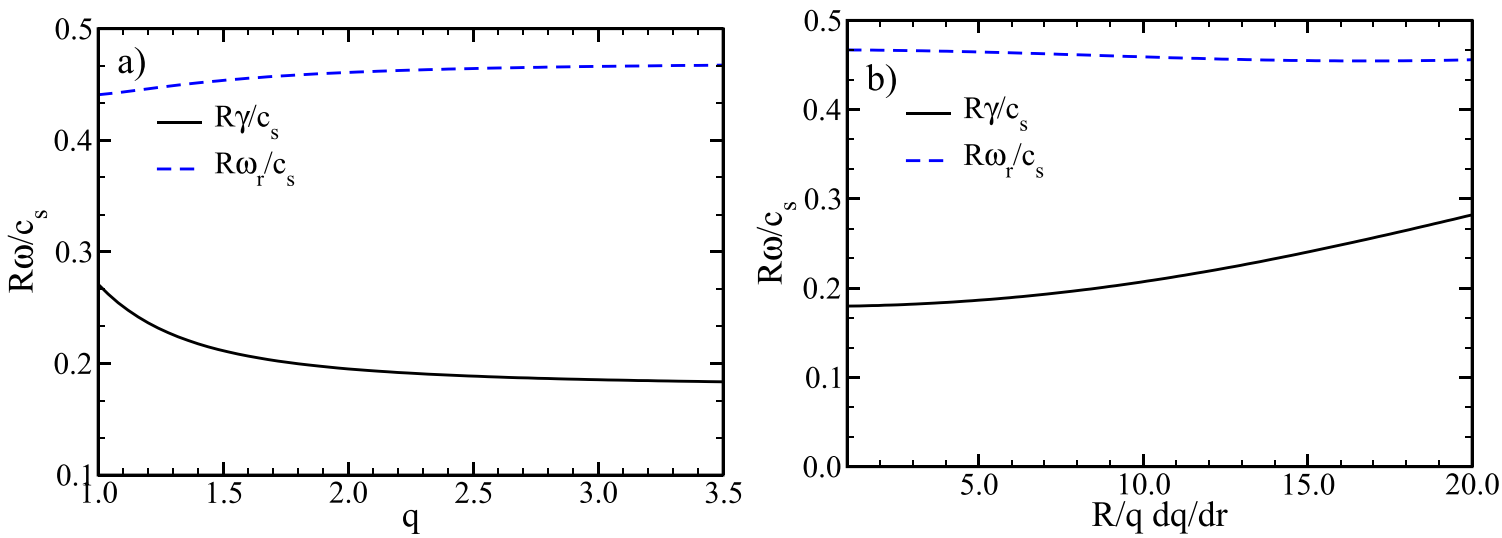

FIG. 7. (a) Normalized growth rate of most unstable microtearing mode and associated normalized real frequency as a function of safety factor, $q$ and (b) as a function of shear, $(R / q) d q / d r$. The plots are obtained for $k_{\mathrm{y}} \rho_{\mathrm{s}}=0.1$, for $k_{\mathrm{x}} \rho_{\mathrm{s}}=1.0$, and for $r_{\mathrm{min}}=0.38 \mathrm{~m}$. The other parameters are the same as those used in Fig. 1.

model. For low values of plasma $\beta_{\mathrm{e}}$, the growth rate and real frequency both increase with increasing $\beta_{\mathrm{e}}$ but then both saturate for higher values of $\beta_{\mathrm{e}}$.

The normalized growth rate of the most unstable microtearing mode and the associated real frequency are shown as a function of the safety factor, $q$, in Fig. 7(a), and as a function of magnetic shear, $(R / q) d q / d r$, in Fig. 7(b). Note that these results are obtained for $r_{\min }=0.38 \mathrm{~m}$, whereas in Figs. $1-6$, results are presented for $r_{\min }=0.5 \mathrm{~m}$. The safety factor and shear both enter in the calculation of parallel wavevector, $k_{\|}$. The safety factor also enters in the average curvature formula. The increase in the value of the safety factor is found to be stabilizing for the microtearing mode, whereas the increase in the value of the magnetic shear is found to be destabilizing. This is in contrast to the ITG and TEM modes where the increase in the magnetic shear is found to be stabilizing. The destabilization of microtearing modes with the increase in magnetic shear can be used to distinguish microtearing modes from other drift modes like ITG and TEM modes.
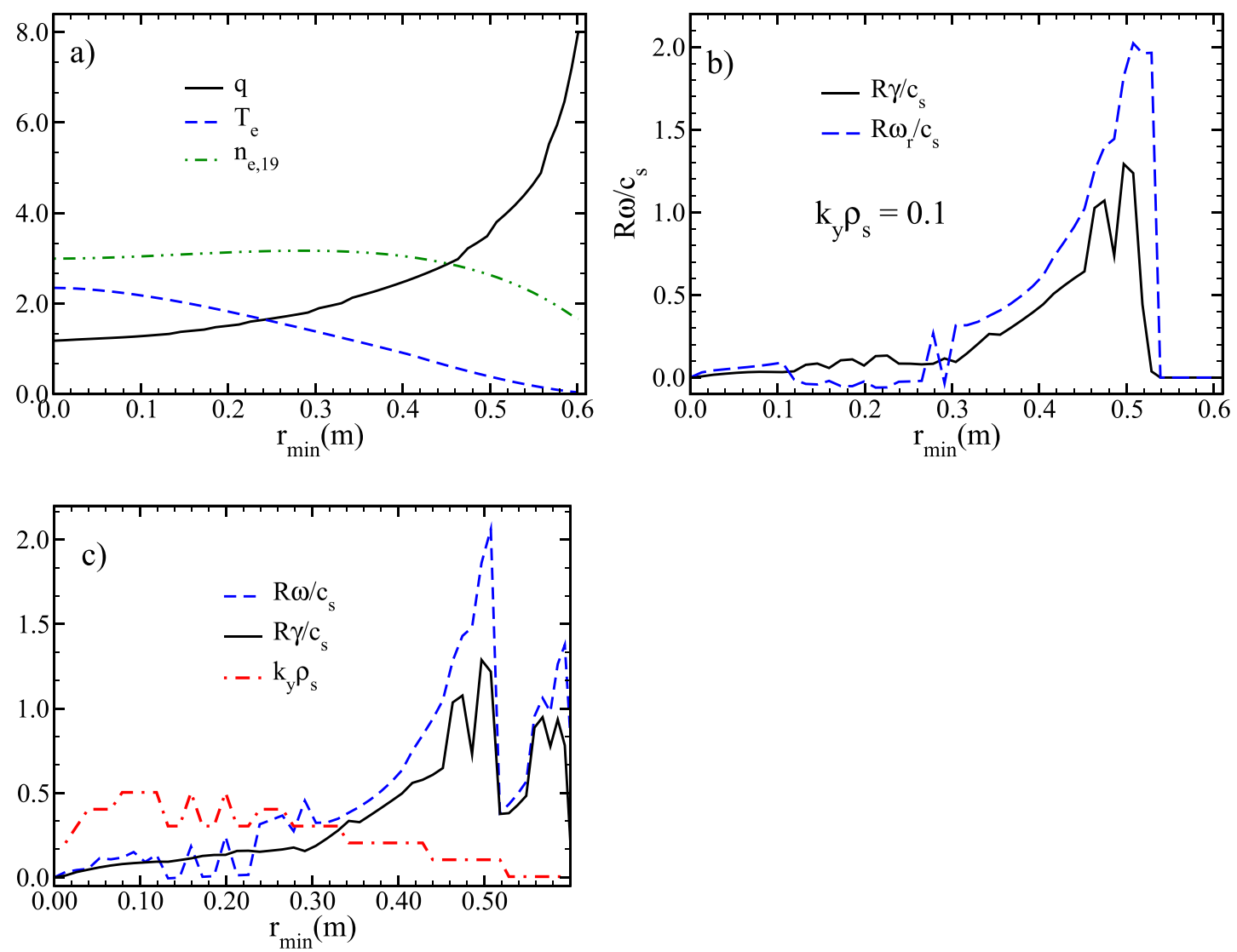

FIG. 8. (a) The equilibrium DIII-D L-mode tokamak profiles, magnetic- $q$, electron temperature $\left(T_{\mathrm{e}}\right)$, and electron density $\left(n_{\mathrm{e}, 19}\right)$. (b) Normalized microtearing mode growth rate of most unstable mode, $R \gamma / c_{\mathrm{s}}$ and associated normalized microtearing mode real frequency, $R \omega / c_{\mathrm{s}}$. (c) The values of $k_{\mathrm{y}} \rho_{\mathrm{s}}$ associated with each microtearing mode growth rate and real frequency are plotted as function of radius. 

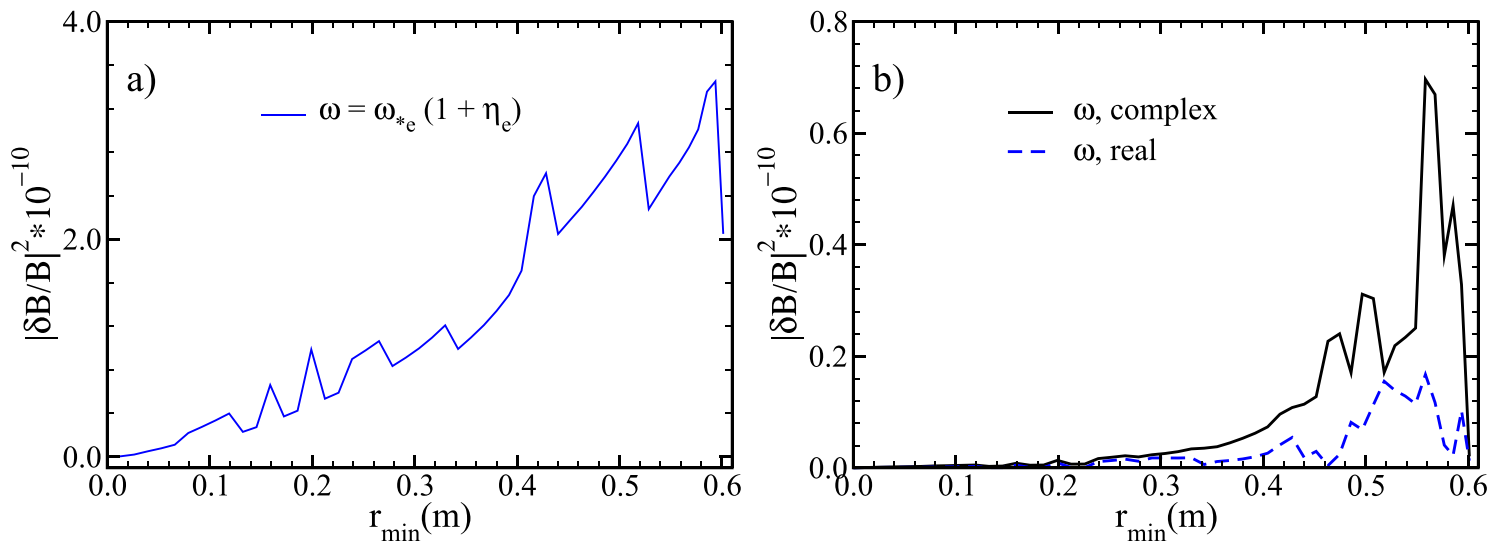

FIG. 9. (a) The normalized magnetic fluctuations are plotted as a function of radius for (a) $\omega=\omega_{* e}\left(1+\eta_{\mathrm{e}}\right)$, (b) $\omega=\omega_{\mathrm{r}}$ (dashed line), and for $\omega=\omega_{\mathrm{r}}+i \gamma$ (solid line) for DIII-D L-mode tokamak.

In Fig. 8(a), the magnetic-q, electron temperature, and electron density profiles are plotted for a DIII-D L-mode tokamak discharge. In Fig. 8(b), the normalized wavenumber, $k_{\mathrm{y}} \rho_{\mathrm{s}}$, is set equal 0.1 and for each value of $r_{\min }$, the DIII-D equilibrium values are used in obtaining the maximum value of the most unstable microtearing mode and the associated real frequency. For Fig. 8(c), the constraint of $k_{\mathrm{y}} \rho_{\mathrm{s}}=0.1$ is removed and for each value of $r_{\text {min }}$, a scan over $k_{\mathrm{y}} \rho_{\mathrm{s}}$ is carried out to determine the value of $k_{\mathrm{y}} \rho_{\mathrm{s}}$ associated with the most unstable mode. The value obtained for $k_{\mathrm{y}} \rho_{\mathrm{s}}$ as well as the normalized maximum growth rate and associated normalize real frequency are plotted in Fig. 8(c) as a function of $r_{\min }$.

In contrast to the fixed $k_{\mathrm{y}} \rho_{\mathrm{s}}=0.1$ case shown in Fig. 8(b), the microtearing modes are found to be unstable even in the edge region $r_{\text {min }} \geq 0.53$ for the values of $k_{\mathrm{y}} \rho_{\mathrm{s}}$ associated at each radius with the most unstable microtearing mode. The values of $k_{\mathrm{y}} \rho_{\mathrm{s}} \leq 0.5$, associated with the most unstable microtearing mode at each radius, indicate that these unstable modes are ion scale modes over the whole plasma radius including the plasma core region.

\section{B. Magnetic fluctuations and resulting electron thermal diffusivity for L- and $\mathrm{H}$-mode like tokamak discharges}

In Ref. 2, the unstable microtearing modes are assumed to saturate at $|\delta B| / B=\rho_{\mathrm{e}} / L_{\mathrm{Te}}$, the ratio of electron gyroradius to the electron temperature scale length. In contrast, the computation of magnetic fluctuations here makes use of the nonlinear microtearing mode envelope equation [Eq. (4)].

The dependence of the square of the magnetic fluctuation strength due to microtearing mode as a function of radius is plotted in Fig. 9 for DIII-D L-mode discharge. In order to compute the strength of the magnetic fluctuations, the $\omega$ in Eq. (4) is calculated in three different ways: (1) $\omega=\omega_{* e}\left(1+\eta_{\mathrm{e}}\right)$; (2) the real frequency, $\omega_{r}$, is used; and (3) the eigenvalue $\omega=\omega_{\mathrm{r}}+i \gamma$ is utilized. The choice of using $\omega=\omega_{* e}\left(1+\eta_{\mathrm{e}}\right)$ in calculating the magnetic fluctuations was proposed in Ref. 2. Since this frequency is not complex but real, the choice of using only the real part of the complex frequency derived using the microtearing mode model described in Ref. 21 is considered. In comparison, the third choice uses the derived complex frequency.

The magnetic fluctuations in Fig. 9(a) are found in the whole radius for the case of $\omega=\omega_{* e}\left(1+\eta_{\mathrm{e}}\right)$. Moreover, the magnetic fluctuations are found to be increasing toward the edge. In the case of using the real or the complex frequency, the magnetic fluctuations disappear in the core region, $r_{\min } \leq 0.22$. The magnetic fluctuations are only found in the region, $r_{\min } \geq 0.22$. However, the strength of magnetic fluctuations is found to be weaker with the choice of using the
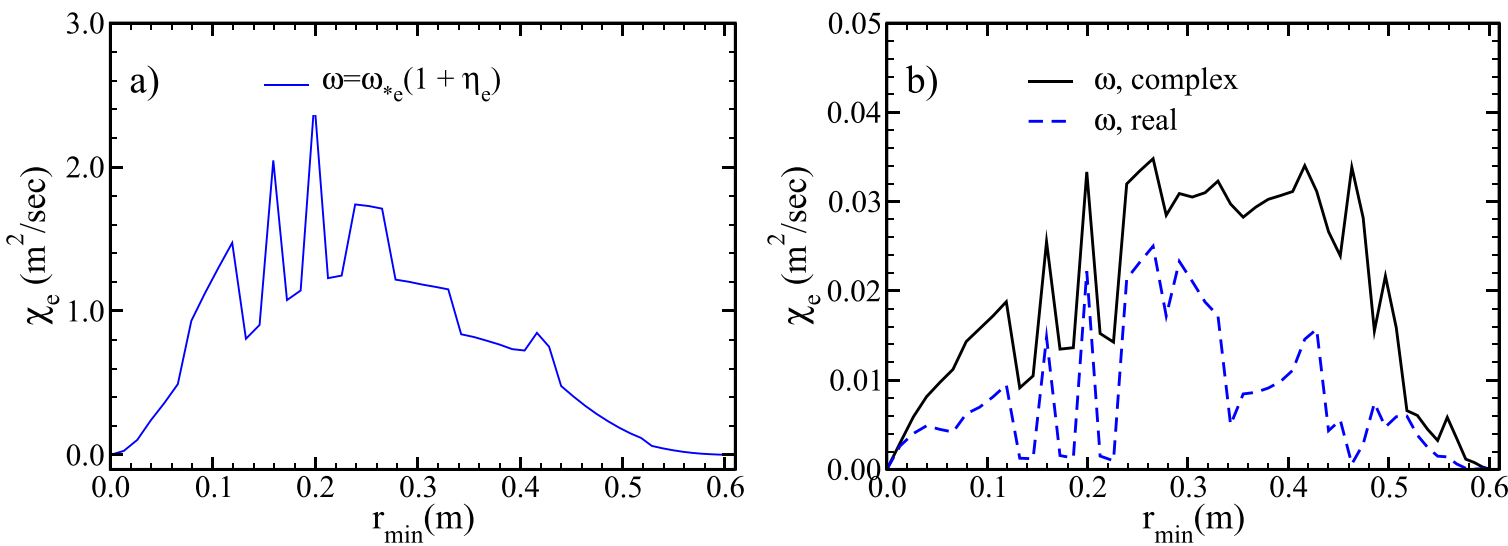

FIG. 10. (a) The electron thermal diffusivity, $\chi_{\mathrm{e}}$ due to the magnetic fluctuations calculated using $\omega=\omega_{* e}\left(1+\eta_{\mathrm{e}}\right),(\mathrm{b}) \omega=\omega_{\mathrm{r}}\left(\mathrm{dashed}\right.$ line), and $\omega=\omega_{\mathrm{r}}+i \gamma$ (solid line) are plotted as a function of radius. 

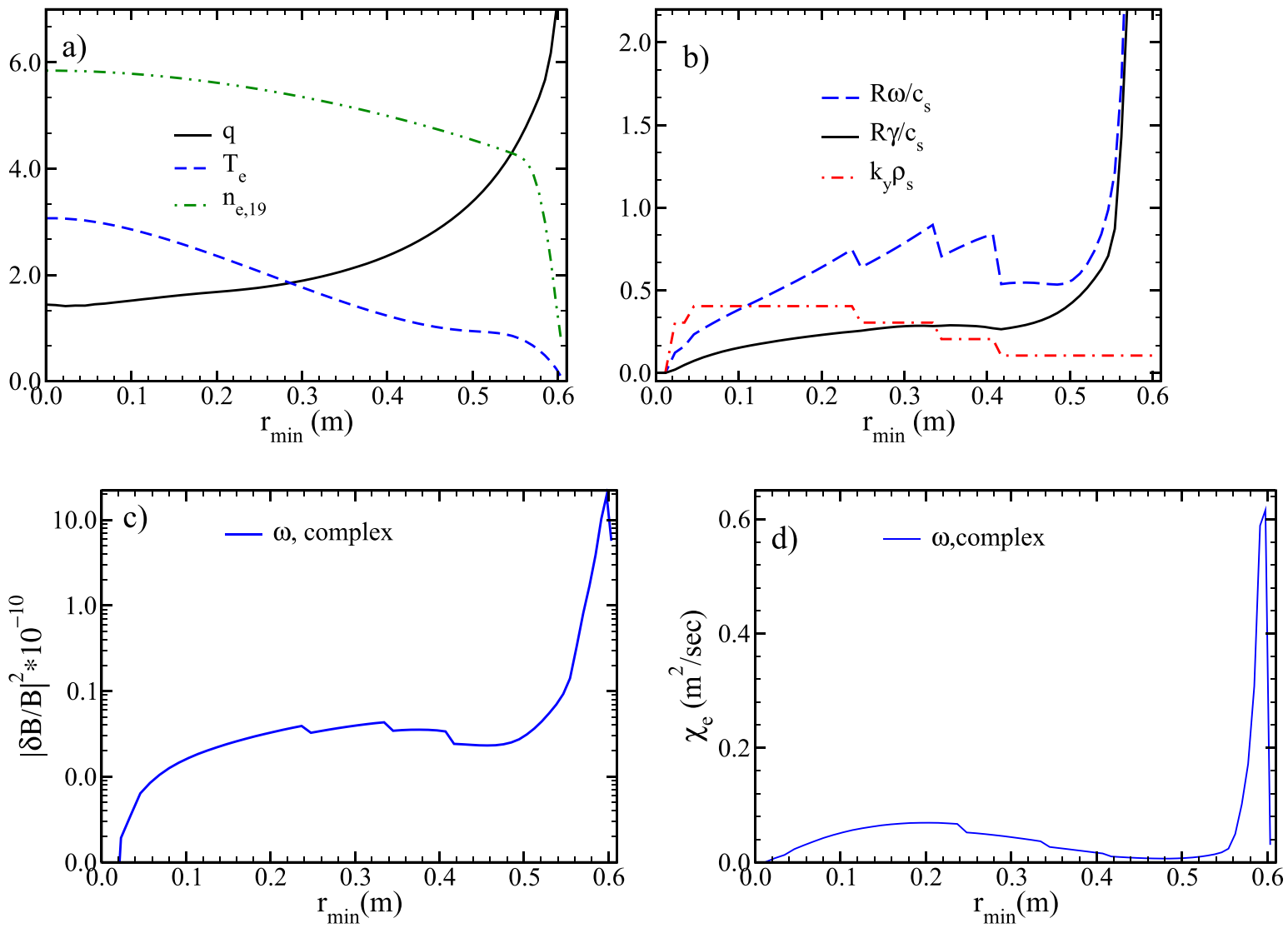

FIG. 11. (a) Equilibrium DIII-D H-mode tokamak profiles, magnetic- $q$, electron temperature $\left(T_{\mathrm{e}}\right)$ and electron density $\left(n_{\mathrm{e}, 19}\right)$. (b) Normalized microtearing mode real frequency, $R \omega / c_{\mathrm{s}}$ (dashed line), normalized microtearing mode growth rate, $R \gamma / c_{\mathrm{s}}$ (solid line), and the corresponding $k_{\mathrm{y}} \rho_{\mathrm{s}}$ (dotted-dashed). (c) Square of a normalized magnetic fluctuation strength. (d) Electron thermal diffusivity $\left(\chi_{\mathrm{e}}\right)$ is plotted as a function of radius for parameters $k_{\mathrm{x}}=5 k_{\mathrm{y}}$.

eigenvalue than using $\omega=\omega_{* e}\left(1+\eta_{\mathrm{e}}\right)$. There is no diagnostic on any tokamak is available at present to measure the internal magnetic field fluctuations and to compare it with the computed magnetic fluctuations.

In Fig. 10, the electron thermal diffusivity, $\chi_{\mathrm{e}}\left(\mathrm{m}^{2} / \mathrm{s}\right)$, due to the saturated microtearing mode is computed as a function of radius by utilizing Eq. (5) for the choice of $\omega=\omega_{* e}\left(1+\eta_{\mathrm{e}}\right), \omega=\omega_{\mathrm{r}}$, and for $\omega=\omega_{\mathrm{r}}+i \gamma$. The $\chi_{\mathrm{e}}$ is found to be small at the edge region of plasma as a result of a large collisionality. The $\chi_{e}$ is found to the order of $0.02 \mathrm{~m}^{2} / \mathrm{s}$ with the choice of $\omega$ being complex value, while it is at the order of $1.0 \mathrm{~m}^{2} / \mathrm{s}$ in the core region of plasma with the choice of $\omega=\omega_{* e}\left(1+\eta_{\mathrm{e}}\right)$.

In Fig. 11(a), the magnetic-q, temperature, and density in DIII-D H-mode tokamak discharge are shown. The microtearing mode normalized real frequency, normalized growth rate, and the corresponding normalized wavenumber, $k_{\mathrm{y}} \rho_{\mathrm{s}}$, are plotted as a function of radius in Fig. 11(b). The whole radius of $\mathrm{H}$-mode discharge is found to be microtearing mode unstable. The growth rate is found to be large in the pedestal region and it is found to be increasing toward the edge. However, the corresponding $k_{\mathrm{y}} \rho_{\mathrm{s}}$ values decrease with increasing radius. The magnetic fluctuations and the thermal diffusivity shown in Figs. 11(c) and 11(d) are found to be large at the top of the temperature and density pedestals. The large values of magnetic fluctuations and diffusivity in the pedestal region can be due to a large temperature and density gradients.

\section{SUMMARY}

Microtearing modes have been identified as a source of significant electron thermal transport in tokamak discharges. A reduced model for microtearing modes in integrated predictive modeling codes is developed. The goal is to improve the prediction of electron thermal transport and, consequently, the prediction of the evolution of the plasma in devices in which microtearing modes have a significant role. A unified fluid/kinetic approach is used in the development of a nonlinear model for the transport driven by microtearing modes.

The dependence of microtearing modes real frequency and growth rate on plasma parameters including wavenumber, collision frequency, temperature gradient, magnetic-q, magnetic shear, density gradient, and plasma beta are presented. The dependence of microtearing mode real frequency, growth rate, magnetic fluctuation, and electron thermal diffusivity on DIII-D L-mode and H-mode plasma is also shown. Most unstable microtearing modes are found for $k_{\mathrm{y}} \rho_{\mathrm{s}} \leq 0.5$, which indicates that microtearing modes are an ion scale electromagnetic instability that is driven by $\eta_{\mathrm{e}}$. Instability threshold in $\eta_{\mathrm{e}}$ is found to be dependent on collision frequency. Modes are found to be stabilizing for both shorter and longer wavelengths due to the effects of field line bending. Large values of $\eta_{\mathrm{e}}$ can be stabilizing for short wavelength modes. The frequency of the microtearing mode generally increases and decreases as the growth rate 
increases and decreases. The dependence of fastest growing microtearing mode on wavenumber with varying collisionality is found to be strong. The most unstable mode in the $k_{\mathrm{y}}$ spectrum shifts toward the lower values of $k_{\mathrm{y}} \rho_{\mathrm{s}}$ with the increase in collision frequency. Microtearing mode growth rate increases with decreasing values of safety factor. The effects of increasing magnetic shear are found to be destabilizing, in contrast to the ITG/TEM modes, where the increase in magnetic shear is stabilizing. The growth rate and real frequency increase with increasing density gradients and plasma $\beta_{\mathrm{e}}$.

The magnetic fluctuation strength which depends upon the most unstable eigenvalues as well as on their sidebands in the $k_{\mathrm{y}}$ spectrum is computed using the L- and H-mode DIII-D profiles. The electron thermal diffusivity that occurs due to the magnetic fluctuations is found to be maximum in the region of moderate collisionality. The magnetic fluctuations and the thermal diffusivity are found to be large at the top of the temperature and density pedestals due to a large value of temperature and density gradients.

A preliminary study of microtearing mode growth rate and real frequency as a function of $k_{\mathrm{y}} \rho_{\mathrm{s}}$, for plasma parameters appropriate for high collisionality NSTX discharges, is being carried out using the reduced MTM transport model. The results obtained are compared and found to be consistent with the microtearing mode results obtained using the gyrokinetic GYRO code. It is planned that these verification results along with other verification and validation results of NSTX discharges will be reported in a future publication.

\section{ACKNOWLEDGMENTS}

This research was supported by the U.S. Department of Energy, Office of Science, under Award Nos. DESC0013977 and DE-FG02-92ER54141.

${ }^{1}$ R. D. Hazeltine, D. Dobrott, and T. S. Wang, "Kinetic theory of tearing instability," Phys. Fluids 18(12), 1778-1786 (1975).

${ }^{2}$ J. F. Drake, N. T. Gladd, C. S. Liu, and C. L. Chang, "Microtearing modes and anomalous transport in tokamaks," Phys. Rev. Lett. 44, 994-997 (1980).

${ }^{3}$ C. M. Roach, D. J. Applegate, J. W. Connor, S. C. Cowley, W. D. Dorland, R. J. Hastie, N. Joiner, S. Saarelma, A. A. Schekochihin, R. J. Akers, C. Brickley, A. R. Field, M. Valovic, and MAST Team, "Microstability physics as illuminated in the spherical tokamak," Plasma Phys. Controlled Fusion 47(12B), B323 (2005).

${ }^{4}$ D. Applegate, C. Roach, J. Connor, S. Cowley, W. Dorland, R. Hastie, and N. Joiner, "Microtearing modes in the mega ampere spherical tokamak," Plasma Phys. Controlled Fusion 49, 1113 (2007).

${ }^{5}$ D. Dickinson, C. M. Roach, S. Saarelma, R. Scannell, A. Kirk, and H. R. Wilson, "Kinetic instabilities that limit $\beta$ in the edge of a tokamak plasma: A picture of an H-Mode pedestal," Phys. Rev. Lett. 108, 135002 (2012).
${ }^{6}$ F. M. Levinton, H. Yuh, M. G. Bell, R. E. Bell, L. Delgado-Aparicio, M. Finkenthal, E. D. Fredrickson, D. A. Gates, S. M. Kaye, B. P. LeBlanc, R. Maingi, J. E. Menard, D. Mikkelsen, D. Mueller, R. Raman, G. Rewoldt, S. A. Sabbagh, D. Stutman, K. Tritz, and W. Wang, "Transport with reversed shear in the National Spherical Torus experiment," Phys. Plasmas 14(5), 056119 (2007).

${ }^{7}$ K. Wong, S. Kaye, D. Mikkelsen, J. Krommes, K. Hill, R. Bell, and B. LeBlank, "Microtearing instabilities and electron transport in the NSTX spherical tokamak," Phys. Rev. Lett. 99, 135003 (2007).

${ }^{8}$ K. L. Wong, S. Kaye, D. R. Mikkelsen, J. A. Krommes, K. Hill, R. Bell, and B. LeBlanc, "A quantitative account of electron energy transport in a National Spherical Tokamak experiment plasma," Phys. Plasmas 15(5), 056108 (2008)

${ }^{9}$ D. R. Smith, W. Guttenfelder, B. P. LeBlanc, and D. R. Mikkelsen, "Identification of microtearing modes below the ion gyroscale in the National Spherical Torus experiment," Plasma Phys. Controlled Fusion 53(3), 035013 (2011).

${ }^{10}$ W. Guttenfelder, J. Candy, S. M. Kaye, W. M. Nevins, E. Wang, R. E. Bell, G. W. Hammett, B. P. LeBlanc, D. R. Mikkelsen, and H. Yuh, "Electromagnetic transport from microtearing mode turbulence," Phys. Rev. Lett. 106, 155004 (2011).

${ }^{11}$ W. Guttenfelder, J. Candy, S. M. Kaye, W. M. Nevins, E. Wang, J. Zhang, R. E. Bell, N. A. Crocker, G. W. Hammett, B. P. LeBlanc, D. R. Mikkelsen, Y. Ren, and H. Yuh, "Simulation of microtearing turbulence in national spherical torus experiment," Phys. Plasmas 19(5), 056119 (2012).

${ }^{12}$ J. Canik, W. Guttenfelder, R. Maingi, T. Osborne, S. Kubota, Y. Ren, R. Bell, H. Kugel, B. LeBlanc, and V. Souhkanovskii, "Edge microstability of NSTX plasmas without and with lithium-coated plasma-facing components," Nucl. Fusion 53(11), 113016 (2013).

${ }^{13}$ S. M. Kaye, W. Guttenfelder, R. E. Bell, S. P. Gerhardt, B. P. LeBlanc, and R. Maingi, "Reduced model prediction of electron temperature profiles in microtearing-dominated National Spherical Torus eXperiment plasmas," Phys. Plasmas 21(8), 082510 (2014).

${ }^{14}$ C. C. Petty, W. Guttenfelder, C. Holland, S. Kaye, J. E. Kinsey, D. C. McDonald, G. R. McKee, L. Vermare, C. Angioni, C. Bourdelle, G. Hoang, F. Imbeaux, F. Ryter, H. Urano, and M. Valovic, "ITER implications of the beta scaling of energy confinement," in Proceedings of the 24th International Conference on Fusion Energy, San Diego, USA, 2012 (IAEA, Vienna, 2012), Paper No. ITR/P1-30.

${ }^{15}$ N. Ohyabu, G. Jahns, R. Stambaugh, and E. Strait, "Correlation of magnetic fluctuations and edge transport in the Doublet III tokamak," Phys. Rev. Lett. 58, 120 (1987).

${ }^{16}$ J. Kesner and S. Migliuolo, "Microtearing modes and fast edge fluctuations in Alcator C-Mod plasmas," Nucl. Fusion 39, 163 (1999).

${ }^{17}$ S. Moradi, I. Pusztai, W. Guttenfelder, T. Fülöp, and A. Molln, "Microtearing modes in spherical and conventional tokamaks," Nucl. Fusion 53(6), 063025 (2013).

${ }^{18}$ H. Doerk, F. Jenko, T. Grler, D. Told, M. J. Pueschel, and D. R. Hatch, "Gyrokinetic prediction of microtearing turbulence in standard tokamaks," Phys. Plasmas 19(5), 055907 (2012).

${ }^{19}$ R. R. Dominguez, M. Rosenberg, and C. S. Chang, "Nonlinear theory of high-m tearing modes," Phys. Fluids 24(3), 472 (1981).

${ }^{20}$ G. G. Craddock and P. W. Terry, "Thermally driven edge magnetic turbulence," Phys. Fluids B 3(12), 3286 (1991).

${ }^{21}$ T. Rafiq, J. Weiland, A. H. Kritz, L. Luo, and A. Y. Pankin, "Microtearing modes in tokamak discharges," Phys. Plasmas 23, 062507 (2016).

${ }^{22}$ T. Rafiq, A. H. Kritz, J. Weiland, A. Y. Pankin, and L. Luo, "Physics basis of multi-mode anomalous transport module," Phys. Plasmas 20(3), 032506 (2013). 